\title{
Mapping the cells in the liver - unchartered subtypes and heterogeneity
}

An in-depth
analysis
revealed
subtypes of
endothelial
cells, Kupffer
cells, immune
cells and
cholangiocytes
that had
previously not
been identified

A human liver cell atlas has been generated by performing single-cell RNA sequencing (scRNA-seq) of $\sim 10,000$ cells from normal liver tissue. This atlas has revealed heterogeneous cell populations and previously unknown cell subtypes in the liver, providing a powerful resource for the analysis of cell types in healthy and diseased livers.

Chronic liver disease (such as viral hepatitis) and hepatocellular carcinoma (HCC) are global health problems. The liver has remarkable regenerative capacity and a high degree of plasticity in liver cells in health and disease. The new research, published in Nature, involved collaboration between basic and clinical scientists. "scRNA-seq provides a unique opportunity for the study of liver disease biology and the identification of novel therapeutic targets," explains Thomas Baumert, a hepatologist whose lab led the translational aspects of the research. Inspired after a sabbatical, he approached Dominic Grün, an expert in quantitative single-cell biology and scRNA-seq, to help build the new liver cell atlas using his group's expertise in the experimental and computational methods needed for the project.

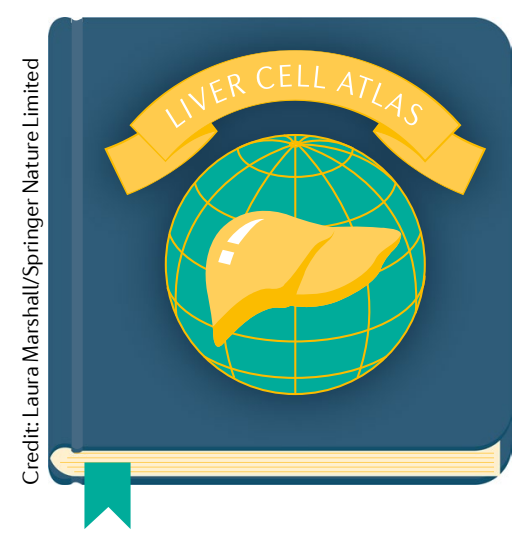

\section{Non-diseased liver tissue} samples were taken from nine human donors, and scRNA-seq was performed on 10,372 cells from these donors to create the human liver cell atlas. An in-depth analysis revealed subtypes of endothelial cells, Kupffer cells, immune cells and cholangiocytes that had previously not been identified. Moreover, transcriptome-wide zonation was observed in hepatocytes and endothelial cells, with evidence that different cell types in the same zone might cooperate and have shared functions. Focussing on cells positive for epithelial cell adhesion molecule (EPCAM), which have been considered as hepatic stem cells, the researchers searched for genuine liver progenitor cells by sorting and then sequencing $\mathrm{EPCAM}^{+}$cells. They found that the $\mathrm{EPCAM}^{+}$cell population was transcriptionally heterogeneous, consisting of a hepatocyte-biased population, cholangiocytes and a naive bipotent progenitor population, and validated their predictions using organoid culture.

Importantly, the human liver cell atlas could be interrogated and used as a reference to examine cell states during disease, in this instance liver cancer. Cells from HCC tissue from three patients were sequenced using scRNA-seq to reveal tumour-associated changes in cancer cells, endothelial cells and immune cells (such as natural killer cells) in comparison to the normal human liver cell atlas. Cancer-specific gene signatures alongside perturbed cellular phenotypes were observed.

Finally, the researchers used scRNA-seq to examine the gene expression signature of human liver cells engrafted in a humanized mouse model. When compared with the human liver cell atlas, differential gene expression was observed between engrafted and non-engrafted human liver cells (including upregulation of WNT and Hedgehog signalling), which could have implications for the interpretation of research using chimeric mouse models.

"Our human liver cell atlas is the most detailed single-cell resolution map of the normal liver to date," explain Baumert and Grün. "We discovered novel subtypes of endothelial cells, liver-resident macrophages, immune cells, and cholangiocytes ... [and] revealed genome-wide zonation of gene expression in human hepatocytes and endothelial cells, and provide evidence for functional cooperation across these cell types in the same zone," they add.

Moving forward, the Grün lab plans to investigate the dynamics of human liver cell populations at the single-cell level during disease progression, focussing on the role and function of bile duct cells and epithelial progenitors. In particular, they are interested in exploring what happens during nonalcoholic fatty liver disease progression and crosstalk in the tumour cell microenvironment in HCC and cholangiocarcinoma. The Baumert lab will use the atlas and scRNA-seq technology for target and drug discovery in nonalcoholic steatohepatitis, fibrosis and HCC. Moreover, they plan to use this approach to better understand the progression of viral hepatitis from inflammation to fibrosis and HCC at the single-cell level.

Katrina Ray

ORIGINAL ARTICLE Aizarani, N. et al. A human liver cell atlas reveals heterogeneity and epithelial progenitors. Nature https://doi.org/10.1038/ s41586-019-1373-2 (2019) FURTHER READING Ben-Moshe, S. \& Itzkovitz, S. Spatial heterogeneity in the mammalian liver. Nat. Rev. Gastroenterol. Hepatol. 16, 395-410 (2019) 